\title{
Global culture: A noise-induced transition in finite systems
}

\author{
Konstantin Klemm, ${ }^{1,2, *}$ Víctor M. Eguíluz, ${ }^{1, \dagger}$ Raúl Toral, ${ }^{1}$ and Maxi San Miguel ${ }^{1}$ \\ ${ }^{1}$ Instituto Mediterráneo de Estudios Avanzados (IMEDEA) (CSIC-UIB), E07122 Palma de Mallorca, Spain \\ ${ }^{2}$ The Niels Bohr Institute, Blegdamsvej 17, DK2100 Copenhagen Ø, Denmark
}

(Received 9 May 2002; published 15 April 2003)

\begin{abstract}
We analyze the effect of cultural drift, modeled as noise, in Axelrod's model for the dissemination of culture. The disordered multicultural frozen configurations are found not to be stable. This general result is proven rigorously in $d=1$, where the dynamics is described in terms of a Lyapunov potential. In $d=2$, the dynamics is governed by the average relaxation time $T$ of perturbations. Noise at a rate $r \leqq T^{-1}$ induces monocultural configurations, whereas $r \gtrsim T^{-1}$ sustains disorder. In the thermodynamic limit, the relaxation time diverges and global polarization persists in spite of a dynamics of local convergence.
\end{abstract}

DOI: 10.1103/PhysRevE.67.045101

PACS number(s): 05.50.+q, 87.23.Ge, 05.40.Ca, 89.65.Ef

Concepts and tools developed in the framework of statistical and nonlinear physics have been shown to be useful in identifying general mechanisms behind collective behavior in social dynamics [1]. Beyond the use of equilibrium ideas based on minimization of a potential or optimization methods [2], models of interacting social agents, as, for example, generalized voter models of opinion formation [3], fall within the context of the study of nonequilibrium phase transitions in lattice models [4]. More generally, these are stochastic spatial models also considered in population dynamics or evolutionary biology [5].

Rather independently of this framework, but closely related in spirit, is the model put forward by Axelrod to describe the dissemination of culture among interacting agents in a society (or among societies) [6]. Culture is here defined as a set of individual attributes that are subject to social influence. The agents are placed in the nodes of a square lattice, and the individual attributes of each site are defined by a set of $F$ features, each taking one of $q$ possible traits. The dynamics of social influence takes into account that "the more similar an actor is to a neighbor, the more likely the actor will adopt one of the neighbor's traits" [6]. Specifically, two neighboring sites chosen at random interact with a probability proportional to the number of common features they already share. An interaction translates into switching one of the different features of one site to the same trait of the neighboring site. In principle, this dynamics tends to homogenize neighbors, and hence to reach a completely ordered state (a monocultural state) in which each feature has the same trait in all lattice sites. What Axelrod emphasizes about his model is that it illustrates how the mechanism of local convergence can generate global polarization. He finds that the system gets trapped in multicultural states with a number of different stable homogeneous domains, called cultural regions. The number of domains in this disordered state is used as a measure of cultural diversity. The quantitative statistical mechanics analysis partially modifies Axelrod's

\footnotetext{
*Present address: Interdisciplinary Centre for Bioinformatics, University of Leipzig, Kreuzstr. 7b, D-04103 Leipzig, Germany. Email address: klemm@izbi.uni-leipzig.de

†Email address: victor@imedea.uib.es
}

conclusion [8]. Defining an order parameter as the relative size of the largest homogeneous domain, one finds a nonequilibrium order-disorder phase transition controlled by the number of traits $q$, which measures the initial degree of disorder of a random configuration. Symmetry breaking leading to dominance of a given culture occurs for $q<q_{c}$, while the order parameter vanishes for $q>q_{c}$. Therefore local convergence is efficient for $q<q_{c}$ and global polarization only persists for $q>q_{c}$.

The analysis of Ref. [8] corresponds to a zero-temperature dynamics in which fluctuations are neglected. Here we address the issue of the effects of random perturbations (noise) following the original suggestion of Axelrod, "Perhaps the most interesting extension and, at the same time, the most difficult one to analyze is cultural drift [modeled as spontaneous change in a trait]" $[6,9]$. Axelrod also identified that a crucial question in this case is to determine a characteristic time scale of the dynamics. We find that this characteristic time scales with the system size. A $q$-independent noiseinduced transition [10] occurs when the noise rate $r$ is of the order of the inverse of this time scale $T$ : for $r \lesssim T^{-1}$ noise induces ordered monocultural configuration because the disordered multicultural states are not stable; for $r \gtrsim T^{-1}$ symmetry restoring by noise is efficient and the disordered multicultural state prevails. In the thermodynamic limit of large systems the condition $r \gtrsim T^{-1} \rightarrow 0$ is satisfied for any finite $r$. In this limit, the fundamental idea of local convergence generating global polarization is recovered.

The model we study is defined [6] by considering $N$ agents as the sites of a lattice. The state of agent $i$ is a vector of $F$ components (cultural features) $\left(\sigma_{i 1}, \sigma_{i 2}, \ldots, \sigma_{i F}\right)$. Each $\sigma_{i f}$ is one of the $q$ integer values (cultural traits) $1, \ldots, q$, initially assigned independently and with equal probability $1 / q$. The time-discrete dynamics is defined as iterating the following steps.

(1) Select at random a pair of neighboring lattice sites $(i, j)[11]$.

(2) Calculate the overlap (number of shared features) $l(i, j)=\sum_{f=1}^{F} \delta_{\sigma_{i f}, \sigma_{j f}}$.

(3) If $0<l(i, j)<F$, the bond is said to be active and sites $i$ and $j$ interact with probability $l(i, j) / F$. In case of interaction, choose $g$ randomly such that $\sigma_{i g} \neq \sigma_{j g}$ and set $\sigma_{i g}$ $=\sigma_{j g}$. 


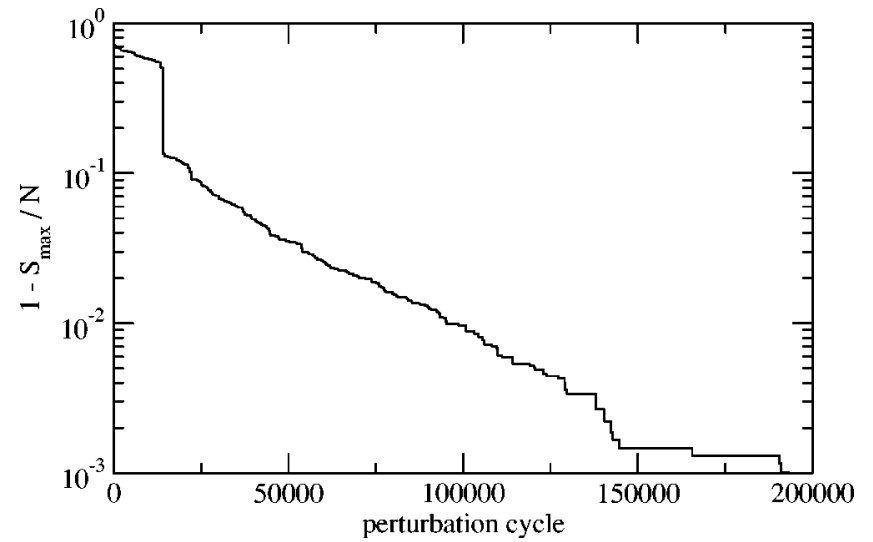

FIG. 1. Lack of stability of disordered absorbing configurations. The order parameter $S_{\max }$ normalized with system size $N$ is shown as a function of perturbation cycles for a square lattice of $N$ $=100^{2}$ sites using $F=10$ and $q=60$.

In any finite lattice the dynamics settles into an absorbing state, characterized by the absence of active bonds. Obviously all the completely homogeneous states are absorbing. Inhomogeneous states consisting of two or more homogeneous domains interconnected by bonds with zero overlap are absorbing as well.

First we consider the question about the stability of these absorbing states. Before we turn to the original twodimensional geometry of the model, we consider the onedimensional lattice with nearest-neighbor interaction. For this geometry the total negative overlap $V$ is a Lyapunov function: a function that never increases during the dynamical process. There is a multiplicity of $q^{F}$ ground states of $V$ that correspond to the homogeneous (ordered) configurations $\sigma_{i f}=\sigma_{j f} \forall i, j$, and $f$, where the potential $V$ takes its absolute minimum $V_{\min }=-N F$. Any other (disordered) absorbing configuration is not the absolute minimum of $V$.

We have not found a parallel rigorous result for $d$-dimensional lattices $(d>1)$. However, by extensive numerical simulations we have verified the lack of stability of disordered configurations for $d=2$ with nearest-neighbor interaction as well. The absorbing states are subject to single feature perturbations, defined as randomly choosing $i$ $\in\{1, \ldots, N\}, f \in\{1, \ldots, F\}$ and $s \in\{1, \ldots, q\}$ and setting $\sigma_{i f}$ $=s$. Then the simulations are designed as follows.

(I) Draw a random initial configuration.

(II) Run the dynamics by iterating steps (1), (2), and (3), until an absorbing state is reached.

(III) Perform a single feature perturbation of the absorbing state and resume at (II).

We find that by these cycles of relaxation (step II) and perturbation (step III) the system is driven to complete order, where $\sigma_{i f}=\sigma_{j f}, \forall i, j$, and $f$. Thus, as in the onedimensional case, only the completely ordered configurations are stable, all other absorbing configurations are merely metastable. In Fig. 1 we have plotted a typical evolution of the order parameter considered in Ref. [8], that is, the size of the largest homogeneous domain $S_{\max }$. We observe that, although eventually its value could decrease, $S_{\max }$ typically increases in each cycle of perturbation and relaxation [13].

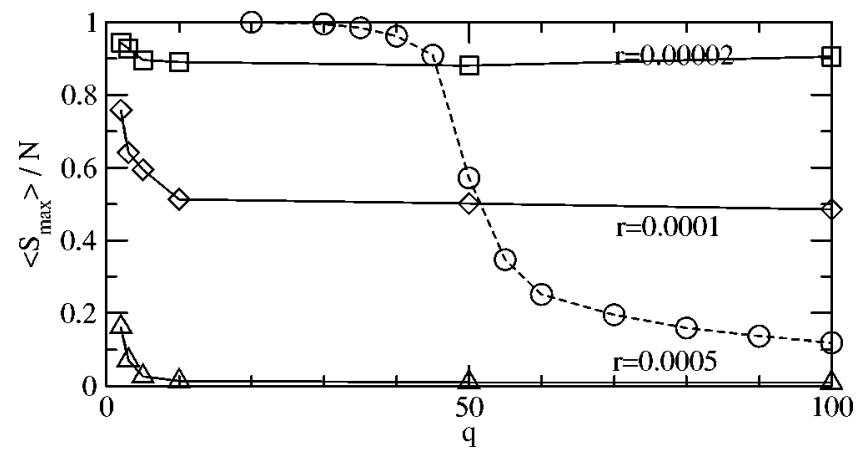

FIG. 2. $\left\langle S_{\max }\right\rangle$ as a function of $q$ for different values of $r$. The dashed line is for $r=0$ with a transition at $q=q_{c} \approx 50$. The simulations used $N=50^{2}$ sites with $F=10$ features. Values $\left\langle S_{\max }\right\rangle$ in Figs. $2,3,4$, and 5 are averages over 500 configurations. Measurements were taken after a relaxation time of $100 N^{2}$ time steps.

We next consider the effect of cultural drift modeled as a random perturbation acting continuously on the system (noise). This is implemented by including a fourth step in the iterated loop of the model.

(4) With probability $r$, perform a single feature perturbation.

Note that now the cultural imitation among sites and the perturbations are two processes acting on the state vectors on time scales separated by the factor $r$. In contrast to the previous scenario, the system is not necessarily in an absorbing configuration when a perturbation occurs.

Figure 2 shows the average order parameter $\left\langle S_{\max }\right\rangle$ as a function of the number of traits $q$. In the case $r=0$, we observe the expected transition from order to disorder as $q$ is increased $[8,14]$. Strikingly, no such transition is observed for noise rate $r \neq 0$. In fact, $\left\langle S_{\max }\right\rangle$ is practically independent of $q$ except for small values. This becomes clear by noticing that with probability $1 / q$ a perturbation does not change the configuration. Therefore considering the effective noise rate $r^{\prime}=r(1-1 / q)$ the data collapse onto a single curve $\left\langle S_{\max }\left(r^{\prime}\right)\right\rangle$ (see Fig. 3). This curve identifies, for a fixed size of the system, a continuous order-disorder transition controlled by the noise rate. In addition, it shows that the transition has universal scaling properties with respect to the value of $q$.

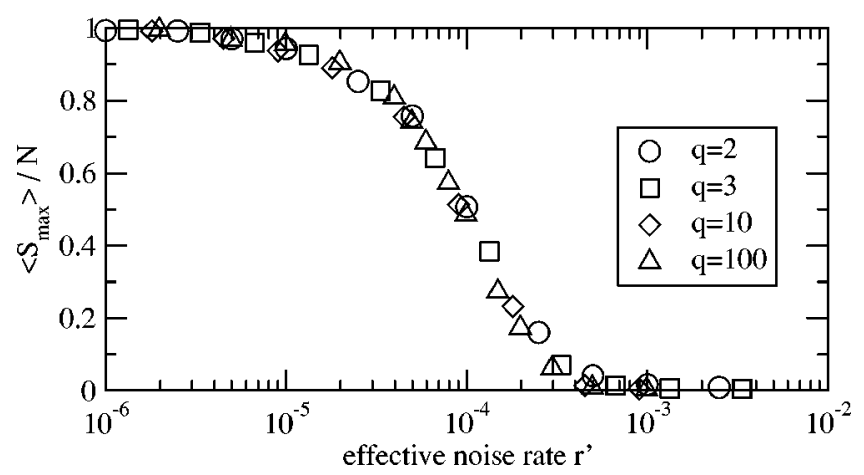

FIG. 3. $\left\langle S_{\max }\right\rangle$ as a function of the effective noise rate $r^{\prime}=r(1$ $-1 / q$ ) for different values of $q$. Simulations have been run in systems of size $N=50^{2}$ with $F=10$. 
It is worth noting that the limit $r \rightarrow 0$ does not recover the results for the original model without noise, corresponding to $r=0$. The fact that for $r \rightarrow 0$ we find an ordered state for all values of $q$ is linked to the "metastable nature" of the inhomogeneous absorbing states previously discussed. Perturbations at a vanishingly small rate are sufficient to allow the system to escape from these states.

The order-disorder transition is also observed in the distribution of sizes of homogeneous domains. For small noise rates typically a single cluster spans the whole system. As the noise rate is increased, smaller clusters become more and more abundant. In the transition region the distribution of domain sizes follows a power law. The exponent is approximately -2 .

What causes the onset of disorder with increasing noise rate? For a sufficiently small noise rate the system has enough time to relax to an absorbing configuration between perturbations. Then the situation would be comparable to the previously studied case of alternating perturbation and relaxation: after a transient of reducing the disorder from the initial condition like the one shown in Fig. 1, the system will spend most of the time in one of the homogeneous configurations. At variance with the case of alternating perturbation and relaxation where the ordered state cannot be left once reached, there will be, on long time scales, random jumps taking the system from a homogeneous state to another homogeneous one. If the noise rate is increased such that the typical time $1 / r$ between perturbations is shorter than the average relaxation time, perturbations are "accumulated" in the system and disorder is built up. According to this picture the average relaxation time $T$ of perturbations of a homogeneous state sets the transition where $r T=O(1)$.

An approximate argument for the calculation of $T$ is as follows. A single feature perturbation of an ordered state at time $t=0$ induces a "damage" of size $x(t=0)=1$ in one of the components. In the following time steps the damage may spread until an ordered state is reached again by $x(t)=0$ or $x(t)=N$. The probability $D_{x}$ for $x$ to increase or decrease is the fraction of active bonds, which again depends on $x$ but also on the current shape of the damage cluster. This complication is avoided in a mean-field description, where a bond exists between any two sites. Then the number of active bonds is simply the number of pairs $(i, j)$ of sites $i$ carrying the damage and sites $j$ not carrying the damage. Given damage size $x$ we find $x(N-x)$ active out of $N(N$ $-1) / 2$ bonds, yielding an update probability (for $N \gg 1$ ): $D_{x}=2 N^{-2} x(N-x)$. The average first exit time, that is the average number of time steps $\tau_{x}$ required to reach an absorbing boundary $(x=0$ or $x=N)$ when starting from $x(t=0)$ $=x$, fulfills the recursion relation [15]

$$
\tau_{x}=\frac{1}{2} D_{x}\left(\tau_{x-1}+\tau_{x+1}\right)+\left(1-D_{x}\right) \tau_{x}+1
$$

with the boundary conditions $\tau_{0}=\tau_{N}=0$. This equation has the solution

$$
\tau_{x}=2 \frac{x}{N} \sum_{\xi=1}^{N-1} \frac{(N-\xi)}{D_{\xi}}-2 \sum_{\xi=1}^{x-1} \frac{(x-\xi)}{D_{\xi}} .
$$

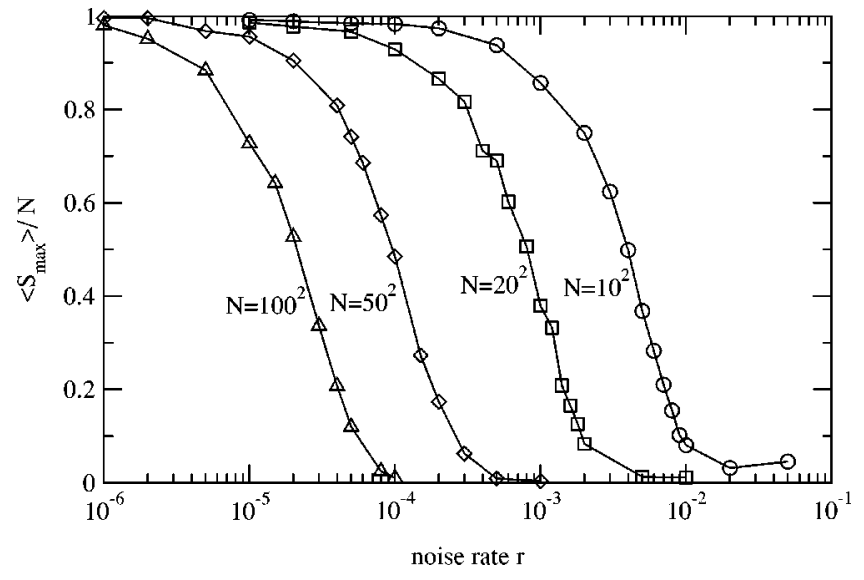

FIG. 4. $\left\langle S_{\max }\right\rangle$ as a function of noise rate for different values of $N$. Simulations were performed with $F=10$ and $q=100$.

The average relaxation time $T$ is the average first exit time for an initial damage size $x=1$. Thus we obtain $T=\tau_{1}$ $=N\left[\gamma+\ln (N-1)+O\left(N^{-1}\right)\right]$, with Euler's constant $\gamma$ $\approx 0.577$. For large $N$ we approximate

$$
T=N \ln (N),
$$

so that the average relaxation time of perturbations diverges with increasing system size [16].

This result, together with our argument that the transition occurs for $r T=O(1)$, suggests to consider systems of different size. We always find a smooth transition from the ordered to the disordered state as $r$ is increased, see Fig. 4. The noise rate necessary to induce disorder is seen to decrease with growing system size. Figure 5 confirms that $T$ is the relevant

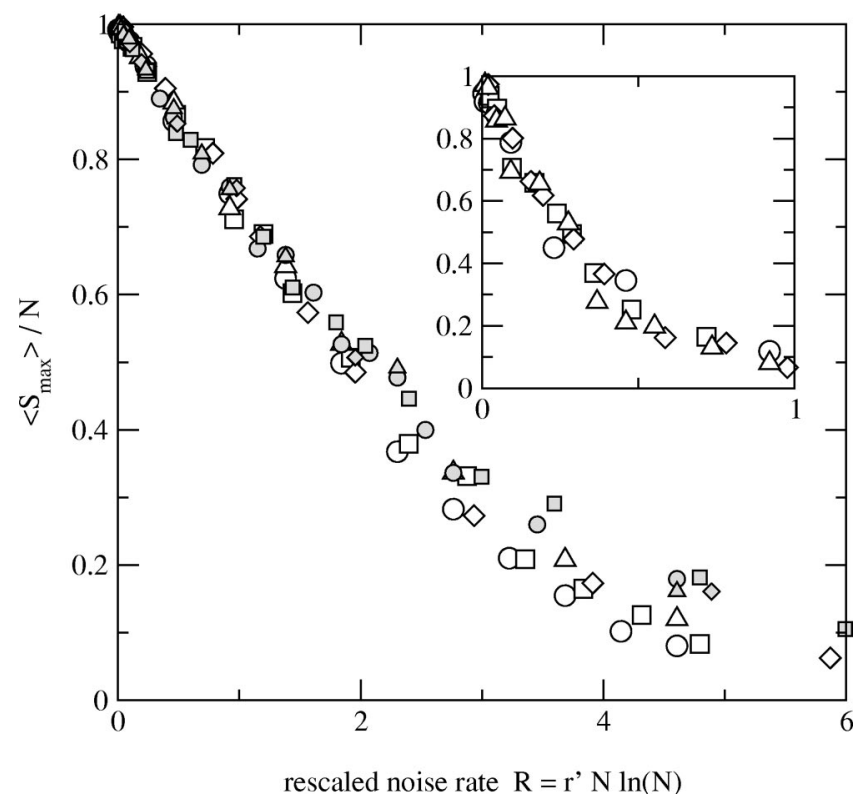

FIG. 5. $\left\langle S_{\max }\right\rangle$ plotted as a function of the rescaled noise rate $R=r N \ln N$. Displayed open symbols and model parameters are the same as in Fig. 4. Filled symbols are for the corresponding case with $q=2$. The inset is the corresponding plot for the decoupled model, see the main text. 
time scale for the onset of disorder. The data of Fig. 4 collapse into a single curve when plotted as a function of a rescaled noise rate $R=r T=r N \ln (N)$ which incorporates noise rate $r$ and system size $N$. Recalling our result for a fixed system size, we conclude that there is a universal scaling form for the order parameter describing the transition: $\left\langle S_{\max }(r, q, N)\right\rangle=\left\langle S_{\max }[f(r, q, N)]\right\rangle \quad$ with $\quad f(r, q, N)=r(1$ $-1 / q) N \ln (N)$.

Note that our analysis assumes that perturbations can always relax to the homogeneous configuration. This assumption is not fulfilled when a sequence of perturbations affects all features of the same site without intermediate relaxation. This introduces nonoverlapping "islands" of sites which do not interact with their neighbors. The effect becomes relevant for very large noise rates and a small number of features (see Fig. 5). It is also relevant for $F=2$ giving a different behavior corresponding to the different type of transition for $r$ $=0[8]$.

We finally note that for the correct prediction of the characteristic time scale $T$ and the change of behavior with noise rate $r$, the dependence of the interaction probability on the sites' overlap need not be taken into account. This motivates the introduction of a "decoupled" version of the dynamics in which a site always adopts the trait of the chosen neighboring site independently of the number of shared features. It turns out that the size of the largest cluster follows the same dependence on the parameters $q, r>0$ and $N$ as in the original model (inset of Fig. 5). This shows that in the presence of noise our main results are insensitive to one of the basic premises of Axelrod's model.

In summary, we have described the scaling properties of an order-disorder transition induced by noise which occurs at a size-dependent value of the noise rate $T^{-1}(N)$. For a finite system and $r \lesssim T^{-1}$ ordered monocultural configurations are induced by noise because disordered multicultural configurations are not stable. In the thermodynamic limit $N \rightarrow \infty$, the condition $r \gtrsim T^{-1}(N) \rightarrow 0$ is satisfied for arbitrarily small noise rate and the system remains in a disordered or multicultural state. In any case, cultural drift changes the nature of the ordered and disordered states. In the ordered state the system is not stuck in a single homogeneous configuration, but during long time scales visits in succession a series of monocultural configurations. Likewise, the disordered state is not a frozen configuration but an evolving state with noise sustained dynamics. Thus, the cultural drift appears to be a relevant variable that drastically modifies the dynamics of Axelrod's cultural model. Future extensions of this model should include the consideration of more complex networks of interaction [17].

We acknowledge financial support from MCyT (Spain) and FEDER (EU) under Project Nos. BFM2000-1108, BFM2001-0341-C02-01, and BFM2002-04474-C02-01.
[1] S. M de Oliveira, P.M.C. de Oliveira, and D. Stauffer, Nontraditional Applications of Computational Statistical Physics (B. G. Teubner, Stuttgart, 1999).

[2] L. Blume, Games Econ. Behav. 5, 387 (1993); 11, 111 (1995).

[3] S. Galam, J. Stat. Phys. 61, 943 (1990); S. Galam, B. Chopard, A. Masselot, and M. Droz, Eur. Phys. J. B 4, 529 (1998).

[4] J. Marro and R. Dickman, Nonequilibrium Phase Transitions in Lattice Models (Cambridge University Press, Cambridge, 1998); I. Dornic, H. Chaté, J. Chave, and H. Hinrichsen, Phys. Rev. Lett. 87, 045701 (2001).

[5] M. Nowak and R. May, Nature (London) 359, 826 (1992); Int. J. Bifurcation Chaos Appl. Sci. Eng. 3, 35 (1993); R. Durret, SIAM Rev. 41, 677 (1999).

[6] R. Axelrod, J. Conflict Res. 41, 203 (1997). Reprinted in Ref. [7].

[7] R. Axelrod, The Complexity of Cooperation (Princeton University Press, Princeton, NJ, 1997).

[8] C. Castellano, M. Marsili, and A Vespignani, Phys. Rev. Lett. 85, 3536 (2000).

[9] See also the discussion about the importance of cultural drift in Ref. [7], pp. 145-147.

[10] We use the term transition to refer to the change of behavior observed in the system, although we will later show that it is not a phase transition in the sense of equilibrium statistical mechanics because it disappears in the thermodynamic limit.

[11] Interactions beyond nearest neighbors have been considered in Refs. [6] and [12].

[12] J.M. Greig, J. Conflict Res. 46, 225 (2002).

[13] The results obtained for single feature perturbations are generic. Qualitatively similar behavior is observed with perturbations affecting simultaneously several features of the selected site. Perturbations randomly assigning new traits to all features of the selected site is a special case.

[14] The model analyzed in Ref. [8] is different from Axelrod's model as in the initial condition the traits are not equally but Poisson distributed. As we employ Axelrod's original model with uniform distribution of traits, our result for $r=0$ confirms that the order-disorder transition also occurs in the original model.

[15] G.R. Grimmett and D.R. Stirzaker, Probability and Random Processes (Oxford Science Publications, Oxford, 1982).

[16] For a one-dimensional lattice there is a similar qualitative behavior. However, the scaling $T \sim N^{2}$ is obtained from Eq. (2), as in this case $D=2 / N$.

[17] K. Klemm, V.M. Eguíluz, R. Toral, and M. San Miguel, Phys. Rev. E 67, 026120 (2003). 